\title{
A Case of Primary Malignant Melanoma of the Larynx
}

\author{
Özge Kaya $^{1 *}$, Dudu Solakoğlu Kahraman ${ }^{1}$, Gülden Diniz ${ }^{1}$, Gönül Demir ${ }^{2}$
}

\begin{abstract}
Objective: Malignant melanoma of the larynx is an uncommon tumor type that can be seen as a primary tumor or as a metastasis from a cutaneous primary lesion. Morphological appearances of this tumor are readily confused with the other types of laryngeal cancers. A new case of primary laryngeal malignant melanoma has been presented in this case report .

Case: The patient was a 54-year-old man without any clinical evidence of other cutaneous malignant melanocytic lesions. Microscopically, polygonal-epithelioid tumor cells containing cytoplasmic and nuclear melanin were located under the mucosal squamous epithelial cells. Atypical mitotic figures were also present. Ulceration and disruption of the epithelium weren't observed. Immunohistochemical studies were positive for S100, HMB-45, Melan-A and vimentin while it was negative for cytokeratin. Based on findings this diagnosis was established.
\end{abstract}

Keywords: Mucosal malignant melanoma, laryngeal primary melanoma, malignant melanoma.

\section{Introduction}

Malignant melanomas are malignant tumors, most of them with cutaneous origin. Compared with cutaneous melanomas of the head and neck, primary melanoma of the upper airways and digestive tract has poor prognosis. In the medical literature only a few cases of mucosal derived malignant melanoma have been reported. Mucosal malignant melanoma of the upper aero digestive tract represents $0.5 \%$ to $3 \%$ of all malignant melanomas while laryngeal melanomas represent up to $3.6-7.4 \%$ (1). Therefore, knowledge about clinicopathologic features, treatment protocols and prognosis of mucosal malignant melanomas are not clearly established.

In this report, we define a case of primary malignant melanoma of the larynx.

\section{Case}

A 54-year-old man was admitted to our hospital with external center biopsy result. At the time of admission, he had suffered recurrent episodes of hoarseness in the past 6 months. He had no other complaints or symptoms. The patient admitted smoking average of 20 cigarettes a day for more than 30 years. He was also an occasional drinker. Computed tomography scan of the larynx demonstrated the presence of $7 \mathrm{~mm}$ diameter nodular lesion in anterior commissure. PET scan of the patient revealed that only larynx show up as bright spots.
After his first diagnosis in a center, he made an application to our laboratory with 4 formalin fixed paraffin-embedded blocks and $4 \mathrm{H} \& \mathrm{E}$ slide preparations. On the routine $\mathrm{HE}$ staining, histopathologic examination larynx mucosal epithelium was intact. Ulceration and disruption of the epithelium weren't observed (Figure 1 and 2). Tumor had a predominantly solid growth pattern infiltrating submucosal compartments and located in the subepithelium. The tumor cells containing cytoplasmic and nuclear melanin, demonstrate pleomorphic eosinophilic cytoplasm with big and round vesicular nuclei with one or many nucleoli (Figure 3). Some areas of the tumor were consisted of spindle-shaped cells, while the others showed polygonal to round epitheloid cells. There was marked cellular pleomorphism, increased mitotic activity. The depth of tumor invasion was $3 \mathrm{~mm}$. Pagetoid spread wasn't observed therefore the tumor was thought to be a nodular melanoma according to these findings. Lymphovascular invasion was not observed.

Immunohistochemical studies were positive for $\mathrm{S} 100$, HMB-45, Melan-A and vimentin while cytokeratin and iron stains were negative (Figures 4). Extensive physical examination by a clinician failed to reveal a primary cutaneous lesion.Finally, the patient was diagnosed with primary malignant melanoma of the larynx. Despite all additional treatments, the patient died 30 months later. 


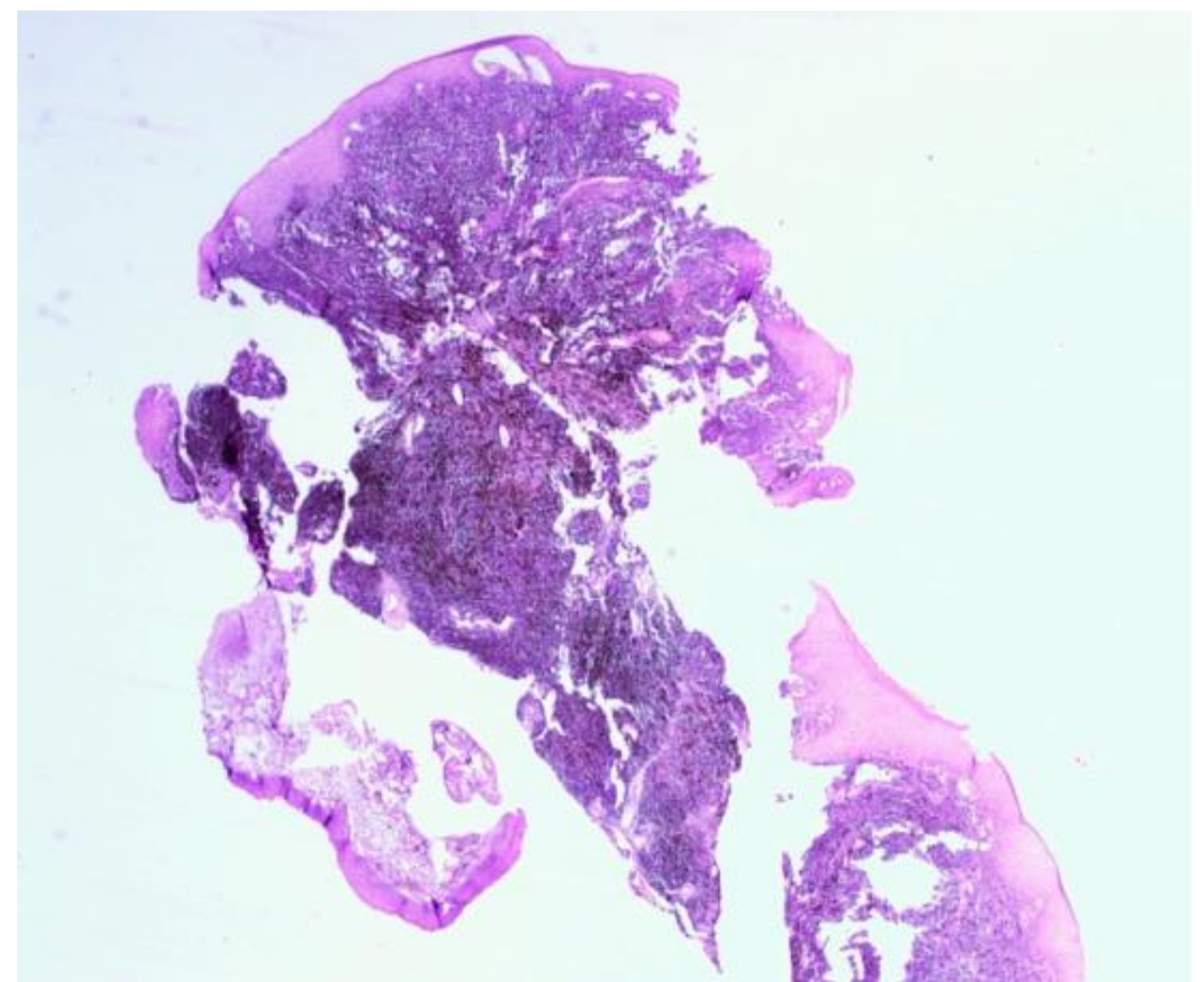

Figure 1: Malignant melanocytes under the epithelium (HE staining 4x)

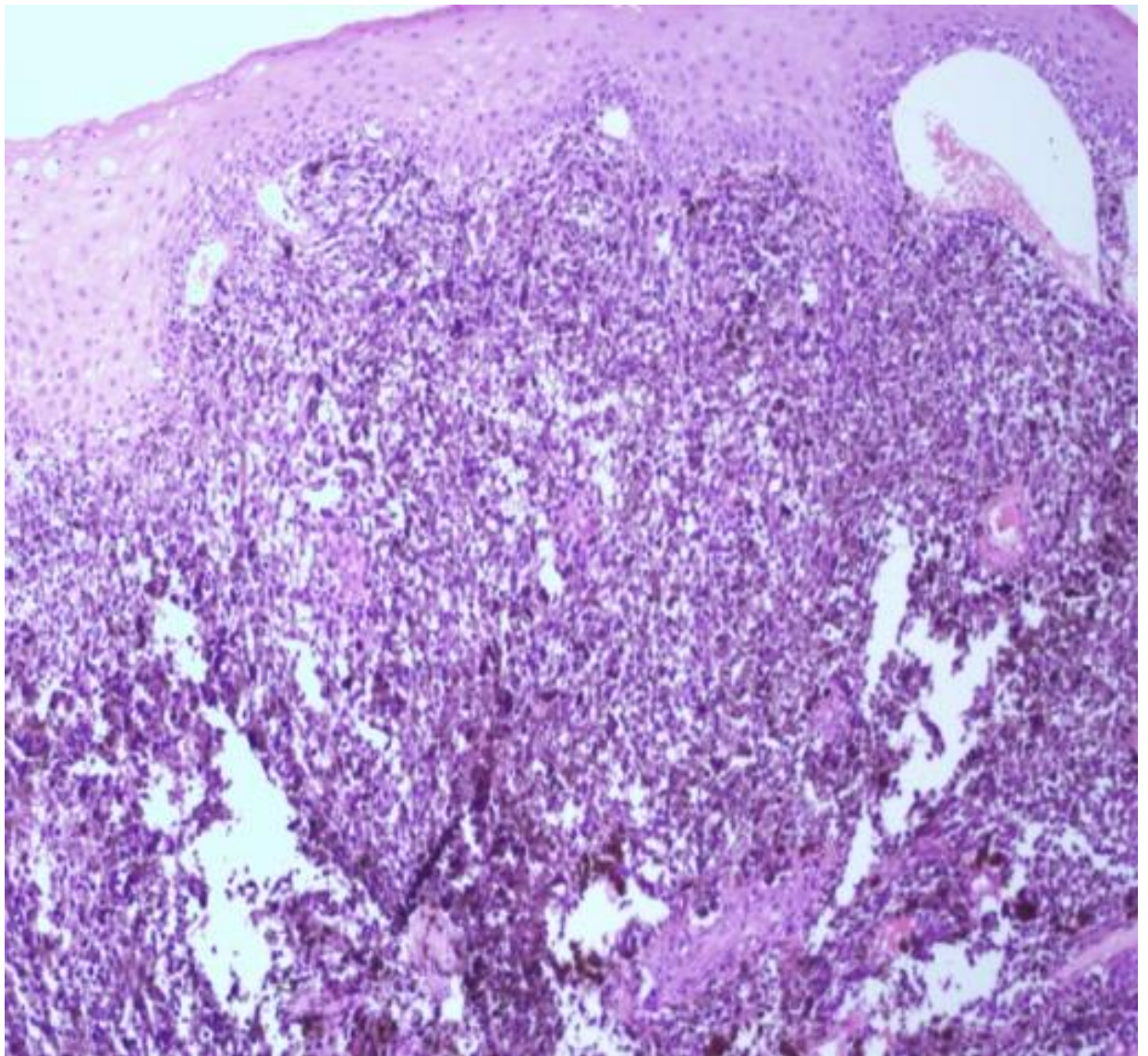

Figure 2: Intact mucosal epithelium, no ulceration (HE staining 10x) 


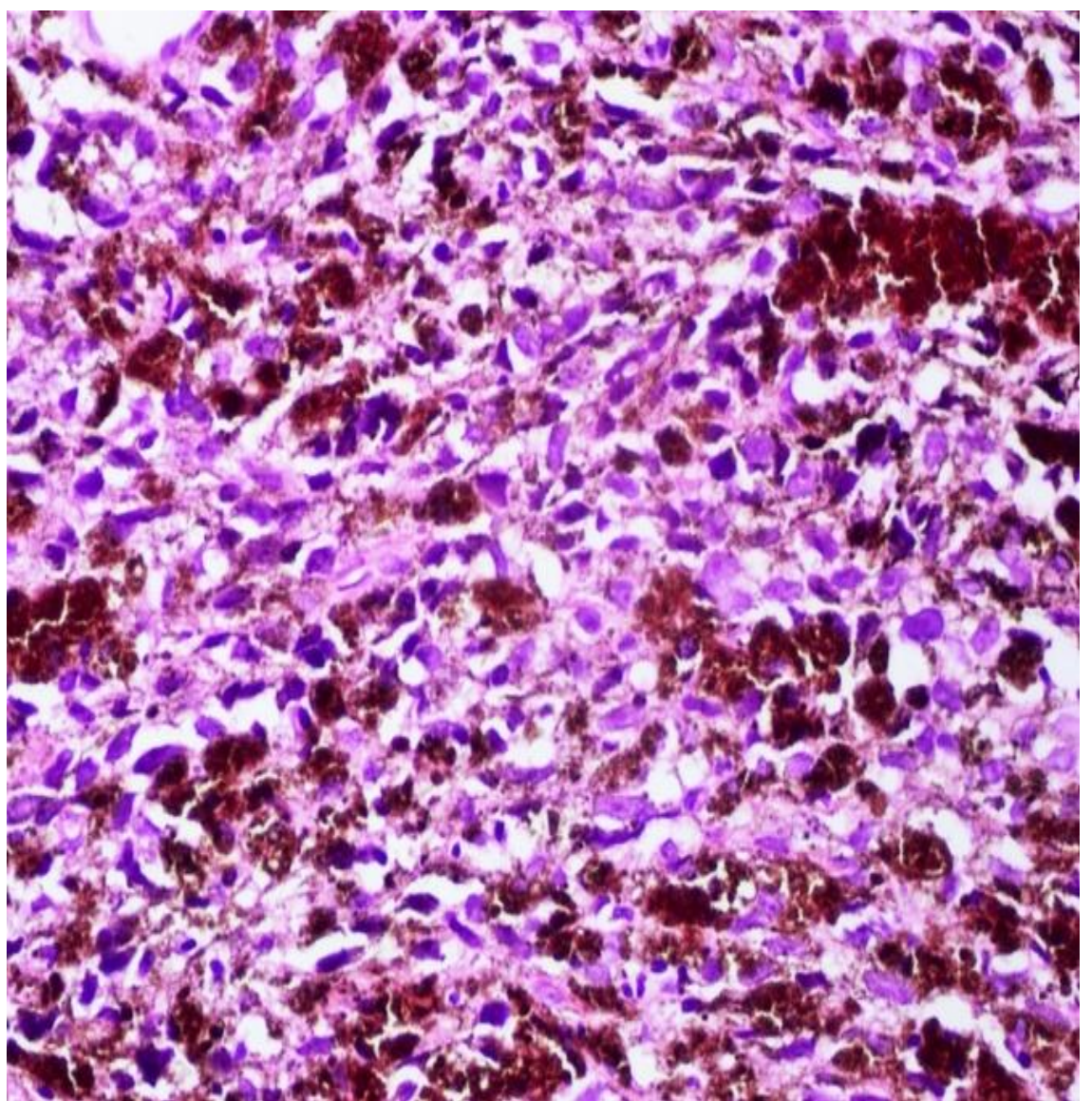

Figure 3: Malignant spindle-shaped cells mixed with pigmented cells (HE staining 40x)

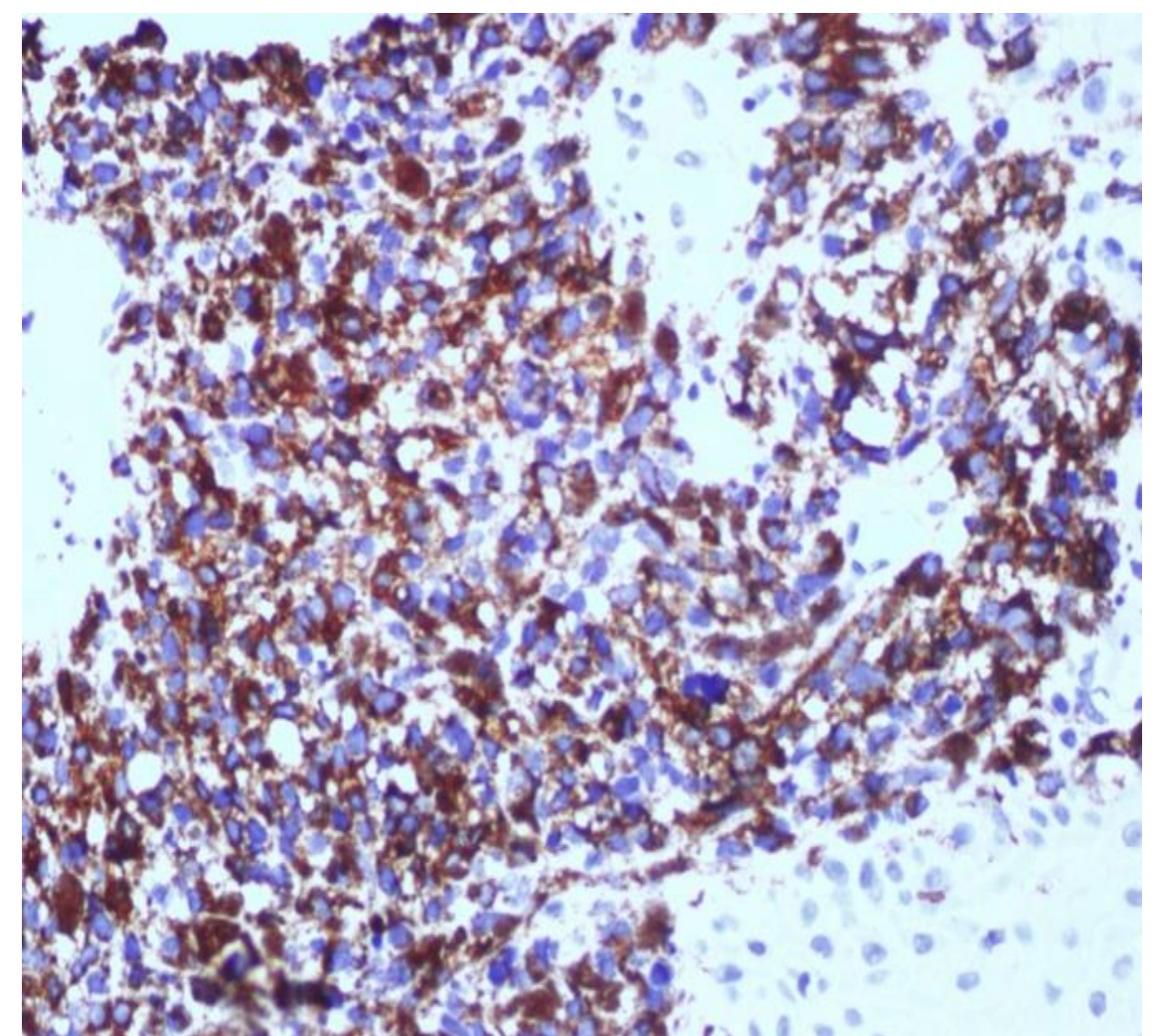

Figure 4: Immunoreaction for HMB-45 showing strong positivity staining of the tumor cell (40x) 


\section{Discussion}

Melanocytes are originate from a neuroectodermal-derived structure called neuronal crest and localize in the cutaneous and non-cutaneous tissue such as leptomeninges, uvea, gastrointestinal, respiratory and genitourinary tracts $(1,2)$. Mucosal melanoma of the larynx is extremely rare tumor with 60 cases reported in the medical literature, but it is more common than metastatic types (2). It is difficult to distinguish primary mucosal melanoma from the metastasis of an unknown cutaneous tumor. Histopathologically, primary melanoma tumors are related to overlying mucosa with junctional activity, but metastatic melanoma do not have any junctional changes with adjacent intact mucosa (6). In this case, mucosa was intact and there was junctional activity between tumor and overlying mucosa. For differentiation of primary lesion from metastasis, revealing a primary cutaneous lesion is also important.

Mucosal melanomas, which are biologically distinct from lesions of cutaneous origin, are caused by unknown factors. Etiological factors, including melanocytosis are still remained speculative (4).

Mucosal melanomas are more aggressive than cutaneous tumors with five-year survival of less than $10 \%$ (5). At the time of diagnosis, having distant metastasis is the cause of poor prognosis (6).

Accurate diagnosis of primary mucosal melanoma of the larynx can be challenging for multiple reasons. Patients may present with non-specific symptoms of hoarseness or hemoptysis. The differential diagnosis of laryngeal masses includes squamous cell carcinoma, neuroendocrine carcinoma, paraganglioma, lymphoma and granulomas. Especially, poorly differentiated squamous cell carcinoma (SCC) of the larynx must be differentiated from melanoma. Melanomas are distinguished from squamous cell carcinoma by its expression of S100, HMB45, melanA and other melanocytic markers (4-7).

The treatment for mucosal melanomas of the head and neck, require complete surgical resection with negative margins (7). Recent studies have shown that postoperative radiation therapy to the affected area may improve the local control, but it is not clear if this translates to an improvement in prognosis (2). Biochemical, targeted therapies and therapeutic drugs directed at genetic mutations are now also under investigation and available for patients (7).

\section{Conclusion}

Primary mucosal malignant melanoma is a rare and highly aggressive tumor. The diagnosis of melanoma can be very challenging for histopathological examination therefore immunohistochemical stains are essential. At the time of diagnosis, any other primary cutaneous lesions need to be excluded.

Conflict of Interest: The authors declare no potential conflicts of interest with respect to the research, authorship, and/or publication of this article.

Author's Contributions: ÖK, DSK, GD, GD: Research concept and design; data collecting, patient examination ÖK: Preparation of article, and Revisions. All authors approved the final version of the manuscript

Ethical issues: All Authors declare, originality and ethical approval of research. Responsibilities of research, responsibilities against local ethics commission are under the Authors responsibilities.

\section{References}

1. Wenig BM. Laryngeal mucosal malignant melanoma: a clinicopathologic,immunohistochemical, and ultrastructural study of four patients and a review of the literature. Cancer. 1995;75:15681577

2. Cojocaru O, Aşchie M, Mocanu L, Baltatescu GI. Laryngeal primary malignant melanoma: a case report.Rom J MorpholEmbryol 2015, 56(4):1513-1516

3. Amin $\mathrm{HH}$, Petruzzeli GJ, Huain AN, Nickoloff BJ. Primary malignant melanoma of the larynx. Arch Pathol Lab Med,2001,125:271-273.

4. El-Naggar AK, Chan JKC, Grandis JR, Takata T, Slootweg PJ. World Health Organization Classification of Head and Neck Tumours. 4th ed. Lyon: IARC; 2017. pp. 60-61. 126-127.

5. Terada T, Saeki N, Toh K, Uwa N, Sagawa K, Mouri T, Sakagami M. Primary malignantmelanoma of the larynx:a case report and litareture review. AurisNasus Larynx, 2007, 34(1):105-110.

6. Zaghi S, Pouldar D, Lai C, Chettri DK. Subglottic presentation of a rare tumor:primary or metastatic? Primary mucosal melanoma of the subglottic larynx. JAMA Otolaryngol Head Neck Surg,2013,139(7):739-740.

7. Lohia S, Boersma I, Lentsch E. Primary malignant melanoma of the larynx- A case report. Otolaryngology Case Reports 4 (2017) 15-17. 